\title{
Operators whose adjoints are quasi $p$-nuclear
}

by

\author{
J. M. Delgado, C. Piñeiro and E. Serrano (Huelva)
}

\begin{abstract}
For $p \geq 1$, a set $K$ in a Banach space $X$ is said to be relatively $p$-compact if there exists a $p$-summable sequence $\left(x_{n}\right)$ in $X$ with $K \subseteq\left\{\sum_{n} \alpha_{n} x_{n}:\left(\alpha_{n}\right) \in B_{\ell_{p^{\prime}}}\right\}$. We prove that an operator $T: X \rightarrow Y$ is $p$-compact (i.e., $T$ maps bounded sets to relatively $p$-compact sets) iff $T^{*}$ is quasi $p$-nuclear. Further, we characterize $p$-summing operators as those operators whose adjoints map relatively compact sets to relatively $p$-compact sets.
\end{abstract}

1. Introduction. In [4, Grothendieck characterized the compact subsets of a Banach space as those sets lying in the closed convex hull of a null sequence. This result aroused interest in the study of sets sitting inside the convex hull of certain classes of null sequences.

In [13], Sinha and Karn introduced the notion of $p$-compact set $(p \geq 1)$. A set $K$ of a Banach space $X$ is relatively p-compact if it is contained in the $p$-convex hull of a $p$-summable sequence $\left(x_{n}\right)$ in $X$, i.e. $K \subset\left\{\sum_{n} \alpha_{n} x_{n}:\left(\alpha_{n}\right)\right.$ $\left.\in B_{\ell_{p^{\prime}}}\right\}$. This notion opens a new approach to the $p$-approximation property. The authors of [13] investigate when the identity map on $X$ can be approximated by finite rank operators on $p$-compact subsets of $X$ and they connect their results with the $p$-approximation properties defined by Saphar [12] and Reinov [10] (which were conceived via the tensor product route). To this end, there is a previous analysis of the ideal $\mathcal{K}_{p}$ of $p$-compact operators (the operators mapping bounded sets to relatively $p$ compact sets) and it is proved that the adjoint of a $p$-compact operator admits a factorization through a subspace of $\ell_{p}$. Using this factorization, a complete norm $\kappa_{p}$ is defined on the ideal $\mathcal{K}_{p}$. It is shown that $\mathcal{K}_{p}$ is contained in the ideal $\Pi_{p}^{d}$ of operators with $p$-summing adjoint [13, Proposition 5.3] and that $\mathcal{K}_{p}(X, Y)$ contains the space $\mathcal{N}_{p}^{d}(X, Y)$ of operators with $p$-nuclear adjoint whenever $Y$ is reflexive (see the remark after [13, Proposition 5.3]).

2010 Mathematics Subject Classification: 47B07, 46B50, 47B10.

Key words and phrases: $p$-compact sets, $p$-compact operator, weakly $p$-compact operator, $p$-summing operator, quasi $p$-nuclear operator, $p$-nuclear operator. 
The aim of this paper is to deepen the study of $\mathcal{K}_{p}$ and its possible applications. In Section 3, we show the close relationship between $p$-compact operators and quasi $p$-nuclear operators. Quasi $p$-nuclear operators, introduced by Persson and Pietsch in [6], are an important tool to obtain results and counterexamples related to the approximation property of order $p$ (see [8]). We prove that an operator is quasi $p$-nuclear iff its adjoint is $p$-compact (Proposition 3.8); in fact, the dual result is also true, which improves Proposition 5.3 in [13]. Another important result of that section is the characterization of $p$-summing operators as those operators whose adjoints map relatively compact sets to relatively $p$-compact sets. In the last section, we deal with the Banach ideal $V_{p}$ of $p$-completely continuous operators (operators mapping relatively weakly $p$-compact sets to relatively $p$-compact sets) and we show that, though $\Pi_{p} \subset \mathcal{V}_{p}$ [13, Proposition 5.4], the inclusion is strict in general for every $p \geq 1$.

2. Preliminaries and notations. Throughout this paper, $X$ and $Y$ will be Banach spaces. As usual, we denote the closed unit ball of $X$ by $B_{X}$, the dual of $X$ by $X^{*}$, and the space of all bounded (linear) operators from $X$ into $Y$ by $\mathcal{L}(X, Y)$. The subspace of $\mathcal{L}(X, Y)$ consisting of all compact (respectively, weakly compact) operators from $X$ into $Y$ is denoted by $\mathcal{K}(X, Y)$ (respectively, $\mathcal{W}(X, Y)$ ).

Given a real number $p \in[1, \infty)$ and an arbitrary set $I, \ell_{p}(I)$ (respectively, $\ell_{\infty}(I)$ ) stands for the Banach space of all scalar functions $\xi$ defined on $I$ satisfying $\sum_{i \in I}\left|\xi_{i}\right|^{p}<\infty$ (respectively, $\sup _{i \in I}\left|\xi_{i}\right|<\infty$ ) endowed with its natural norm. As usual, we write $\ell_{p}$ instead of $\ell_{p}(\mathbb{N})$.

Let $\ell_{p}^{w}(X)$ be the space of all weakly $p$-summable sequences $\left(x_{n}\right)$ in $X$. It is a Banach space with the norm

$$
\left\|\left(x_{n}\right)\right\|_{p}^{w}=\sup _{x^{*} \in B_{X^{*}}}\left(\sum_{n}\left|\left\langle x_{n}, x^{*}\right\rangle\right|^{p}\right)^{1 / p}=\sup _{\left(\alpha_{n}\right) \in B_{\ell_{p^{\prime}}}}\left\|\sum_{n} \alpha_{n} x_{n}\right\| .
$$

The subspace of $\ell_{p}^{w}(X)$ consisting of the (strongly) $p$-summable sequences is denoted by $\ell_{p}(X)$, which is also a Banach space endowed with the norm

$$
\left\|\left(x_{n}\right)\right\|_{p}=\left(\sum_{n}\left\|x_{n}\right\|^{p}\right)^{1 / p} .
$$

We write $\ell_{\infty}(X)$ for the Banach space of all bounded sequences $\left(x_{n}\right)$ in $X$ with the norm

$$
\left\|\left(x_{n}\right)\right\|_{\infty}=\sup _{n}\left\|x_{n}\right\| .
$$

We denote by $c_{0}(X)$ the space of all norm null sequences in $X$, which is a closed subspace of $\ell_{\infty}(X)$ with the above norm. 
In addition to the classical Banach ideals $[\mathcal{L},\|\cdot\|],[\mathcal{K},\|\cdot\|]$ and $[\mathcal{W},\|\cdot\|]$, we deal with the ideals $\left[\Pi_{p}, \pi_{p}\right]$ of all $p$-summing operators and $\left[\mathcal{N}_{p}, \nu_{p}\right]$ of all $p$-nuclear operators. We also consider the injective hull of $\left[\mathcal{N}_{p}, \nu_{p}\right]$, which has been treated in the literature under the name of the Banach ideal of quasi $p$-nuclear operators [6]. We denote this Banach ideal by $Q \mathcal{N}_{p}$. So, an operator $T: X \rightarrow Y$ is quasi p-nuclear iff $j_{Y} \circ T \in \mathcal{N}_{p}\left(X, \ell_{\infty}\left(B_{Y^{*}}\right)\right)$, where $j_{Y}$ is the natural isometric embedding from $Y$ into $\ell_{\infty}\left(B_{Y^{*}}\right)$. It is well known that $T \in Q \mathcal{N}_{p}(X, Y)$ iff there exists a sequence $\left(x_{n}^{*}\right) \in \ell_{p}\left(X^{*}\right)$ such that

$$
\|T x\| \leq\left(\sum_{n}\left|\left\langle x, x_{n}^{*}\right\rangle\right|^{p}\right)^{1 / p}
$$

for all $x \in X$. The quasi $p$-nuclear norm is

$$
\nu_{p}^{Q}(T)=\inf \left\{\left\|\left(x_{n}^{*}\right)\right\|_{p}: 11 \text { holds for all } x \in X\right\}
$$

for all $T \in \mathcal{Q N}_{p}(X, Y)$. If $\mathcal{A}$ is a Banach ideal, then $\mathcal{A}^{d}$ denotes its dual ideal, that is, $\mathcal{A}^{d}(X, Y)=\left\{T \in \mathcal{L}(X, Y): T^{*} \in \mathcal{A}\left(Y^{*}, X^{*}\right)\right\}$.

If $p>1$ and $p^{\prime}=p(p-1)^{-1}$, the map $\Phi_{p}:\left(x_{n}\right) \in \ell_{p}^{w}(X) \mapsto \Phi_{p}\left(x_{n}\right) \in$ $\mathcal{L}\left(\ell_{p^{\prime}}, X\right)$, where $\Phi_{p}\left(x_{n}\right)\left(\alpha_{n}\right)=\sum_{n} \alpha_{n} x_{n}$, is an isometric isomorphism which allows us to identify the spaces $\ell_{p}^{w}(X)$ and $\mathcal{L}\left(\ell_{p^{\prime}}, X\right)$. For $p=1, \ell_{1}^{w}(X)$ is isometrically isomorphic to $\mathcal{L}\left(c_{0}, X\right)$ under the corresponding map $\Phi_{1}$.

The following notions were introduced by Sinha and Karn in [13] trying to extend the characterization of compact sets in Banach spaces as those sets lying inside of the closed convex hull of a norm null sequence [4]. If $p \in[1, \infty)$, the $p$-convex hull of a sequence $\left(x_{n}\right) \in \ell_{p}^{w}(X)$ is

$$
p \text {-co }\left(x_{n}\right)=\Phi_{p}\left(x_{n}\right)\left(B_{\ell_{p^{\prime}}}\right)=\left\{\sum_{n} \alpha_{n} x_{n}:\left(\alpha_{n}\right) \in B_{\ell_{p^{\prime}}}\right\}
$$

( $c_{0}$ instead of $\ell_{p^{\prime}}$ if $p=1$ ). It is clear that the $p$-convex hull of a sequence is an absolutely convex set; if $p>1$, it is also weakly compact so, in particular, norm closed.

A set $K \subset X$ is relatively $p$-compact if there exists a sequence $\left(x_{n}\right) \in$ $\ell_{p}(X)$ such that $K \subset p$-co $\left(x_{n}\right)$. Since $p$-co $\left(x_{n}\right)$ is a relatively compact set when $\left(x_{n}\right) \in \ell_{p}(X)$, relatively $p$-compact sets in $X$ are relatively compact. If compact sets are viewed as $\infty$-compact sets, then it is easy to show that $p$-compact sets are $q$-compact for $1 \leq p<q \leq \infty$. Notice that the convex hull of a relatively $p$-compact set is relatively $p$-compact too.

A set $K \subset X$ is relatively weakly p-compact if there exists a sequence $\left(x_{n}\right) \in \ell_{p}^{w}(X)$ such that $K \subseteq p$-co $\left(x_{n}\right)$. If $p>1$, relatively weakly $p$-compact sets in $X$ are relatively weakly compact. However, $p=1$ is a pathological case: $B_{c_{0}}$ is weakly 1 -compact since $B_{c_{0}}=p$-co $\left(e_{n}\right)$, where $\left(e_{n}\right) \in \ell_{1}^{w}\left(c_{0}\right)$ is the unit vector basis in $c_{0}$. Again, it is a standard argument to prove that weakly $p$-compact sets are weakly $q$-compact for $1<p<q<\infty$. 
Finally, we recall that an operator $T \in \mathcal{L}(X, Y)$ is said to be $p$-compact (respectively, weakly p-compact) if $T\left(B_{X}\right)$ is relatively p-compact (respectively, weakly $p$-compact) in $Y$. The set of $p$-compact (respectively, weakly $p$-compact) operators from $X$ into $Y$ is denoted by $\mathcal{K}_{p}(X, Y)$ (respectively, $\left.\mathcal{W}_{p}(X, Y)\right)$.

3. Main results. The next propositions are the keys to connect $p$ compactness and quasi $p$-nuclearity.

Proposition 3.1. Let $p \in[1, \infty), T \in \mathcal{L}(X, Y)$ and $\left(y_{n}\right) \in \ell_{p}^{w}(Y)$. The following statements are equivalent:

(a) $\left\|T^{*} y^{*}\right\| \leq\left(\sum_{n}\left|\left\langle y_{n}, y^{*}\right\rangle\right|^{p}\right)^{1 / p}$ for all $y^{*} \in Y^{*}$.

(b) $T\left(B_{X}\right) \subseteq \overline{p-\operatorname{co}\left(y_{n}\right)}$.

Proof. $(\mathrm{a}) \Rightarrow(\mathrm{b})$. By contradiction, assume that there exists $x_{0} \in B_{X}$ so that $T x_{0} \notin \overline{p-\operatorname{co}\left(y_{n}\right)}$. As $\overline{p \text {-co }\left(y_{n}\right)}$ is absolutely convex, we can separate $T x_{0}$ and $\overline{p \text {-co }\left(y_{n}\right)}$ strictly by a closed hyperplane; that is to say, there exist $\alpha>0$ and $y^{*} \in Y^{*}$ such that $\left|\left\langle T x_{0}, y^{*}\right\rangle\right|>\alpha$ and $\left|\left\langle y, y^{*}\right\rangle\right|<\alpha$ for all $y \in \overline{p-\operatorname{co}\left(y_{n}\right)}$. Then

$$
\begin{aligned}
\alpha & <\left|\left\langle T x_{0}, y^{*}\right\rangle\right| \leq\left\|T^{*} y^{*}\right\| \\
& \leq\left(\sum_{n}\left|\left\langle y_{n}, y^{*}\right\rangle\right|^{p}\right)^{1 / p}=\sup _{\left(\alpha_{n}\right) \in B_{\ell_{p^{\prime}}}}\left|\left\langle\sum_{n} \alpha_{n} y_{n}, y^{*}\right\rangle\right| \leq \alpha,
\end{aligned}
$$

a contradiction.

(b) $\Rightarrow$ (a). Given $\varepsilon>0$ and $y^{*} \in B_{Y^{*}}$, choose $x \in B_{X}$ such that $\left\|T^{*} y^{*}\right\|<$ $\left|\left\langle x, T^{*} y^{*}\right\rangle\right|+\varepsilon / 2$. Now, take $\left(\alpha_{n}\right) \in B_{\ell_{p^{\prime}}}$ so that $\left\|T x-\sum_{n} \alpha_{n} y_{n}\right\|<\varepsilon / 2$. Then

$$
\begin{aligned}
\left\|T^{*} y^{*}\right\| & <\left|\left\langle x, T^{*} y^{*}\right\rangle\right|+\varepsilon / 2 \\
& \leq\left|\left\langle T x-\sum_{n} \alpha_{n} y_{n}, y^{*}\right\rangle\right|+\left|\left\langle\sum_{n} \alpha_{n} y_{n}, y^{*}\right\rangle\right|+\varepsilon / 2 \\
& <\sum_{n}\left|\alpha_{n}\right|\left|\left\langle y_{n}, y^{*}\right\rangle\right|+\varepsilon \leq\left\|\left(\alpha_{n}\right)\right\|_{p^{\prime}} \cdot\left(\sum_{n}\left|\left\langle y_{n}, y^{*}\right\rangle\right|^{p}\right)^{1 / p}+\varepsilon \\
& \leq\left(\sum_{n}\left|\left\langle y_{n}, y^{*}\right\rangle\right|^{p}\right)^{1 / p}+\varepsilon
\end{aligned}
$$

and letting $\varepsilon \rightarrow 0$ we obtain the conclusion.

Arguing in a similar way, we obtain the dual version of the above result:

Proposition 3.2. Let $p \in[1, \infty), T \in \mathcal{L}(X, Y)$ and $\left(x_{n}^{*}\right) \in \ell_{p}^{w}\left(X^{*}\right)$. The following statements are equivalent: 
(a) $\|T x\| \leq\left(\sum_{n}\left|\left\langle x, x_{n}^{*}\right\rangle\right|^{p}\right)^{1 / p}$ for all $x \in X$.

(b) $T^{*}\left(B_{Y^{*}}\right) \subseteq \overline{p-\operatorname{co}\left(x_{n}^{*}\right)}$.

Remark 3.3. In Proposition 3.1 we can use $p$-co $\left(y_{n}\right)$ instead of $\overline{p \text {-co }\left(y_{n}\right)}$ in case $p>1$. On the other hand, if $p=1$ and $\left(y_{n}\right) \in \ell_{1}(Y)$, we have $\overline{\left\{\sum_{n} \alpha_{n} y_{n}:\left(\alpha_{n}\right) \in B_{c_{0}}\right\}}=\left\{\sum_{n} \alpha_{n} y_{n}:\left(\alpha_{n}\right) \in B_{\ell_{\infty}}\right\}$ and this set is 1compact too. In fact, for $\delta_{n} \rightarrow \infty$ such that $\sum_{n}\left|\delta_{n}\right|\left\|y_{n}\right\|<\infty$, we have the obvious inclusion

$$
\left\{\sum_{n} \alpha_{n} y_{n}:\left(\alpha_{n}\right) \in B_{\ell_{\infty}}\right\} \subset\left\{\sum_{n} \alpha_{n}\left(\delta_{n} y_{n}\right):\left(\alpha_{n}\right) \in B_{c_{0}}\right\} .
$$

Corollary 3.4. Let $T \in \mathcal{L}(X, Y)$. Then the following properties hold:

(I) If $T \in \mathcal{K}_{p}(X, Y)$, then $T^{*} \in Q \mathcal{N}_{p}\left(Y^{*}, X^{*}\right)$.

(II) $T \in \mathcal{Q \mathcal { N }}_{p}(X, Y)$ iff $T^{*} \in \mathcal{K}_{p}\left(Y^{*}, X^{*}\right)$.

In other words, $\mathcal{K}_{p} \subseteq Q \mathcal{Q N}_{p}^{d}$ and $Q \mathcal{N}_{p}=\mathcal{K}_{p}^{d}$.

The converse of Corollary 3.4(I) cannot be deduced directly from Proposition 3.1. Indeed, if $T^{*} \in Q \mathcal{N}_{p}\left(Y^{*}, X^{*}\right)$, then there exists a sequence $\left(y_{n}^{* *}\right) \in$ $\ell_{p}\left(Y^{* *}\right)$ such that $\left\|T^{*} y^{*}\right\| \leq\left(\sum_{n}\left|\left\langle y_{n}^{* *}, y^{*}\right\rangle\right|^{p}\right)^{1 / p}$ for all $y^{*} \in Y^{*}$, and consequently $T\left(B_{X}\right) \subseteq p$-co $\left(y_{n}^{* *}\right)$. In other words, $T \in \mathcal{K}_{p}\left(X, Y^{* *}\right)$ (although $T(X) \subset Y)$. In addition, we will need to deal with the ideal of so-called $\mathcal{N}^{p}$-operators. We recall that $T \in \mathcal{N}^{p}(X, Y)$ if there exist sequences $\left(x_{n}^{*}\right) \in$ $\ell_{p^{\prime}}^{w}\left(X^{*}\right)$ and $\left(y_{n}\right) \in \ell_{p}(Y)$ such that $T$ admits the representation $T=$ $\sum_{n} x_{n}^{*} \otimes y_{n}$ (note that $\mathcal{N}^{p}(X, Y) \subseteq \mathcal{K}_{p}(X, Y)$ ). The norm in this ideal will be denoted by $\nu^{p}$ and is defined by

$$
\nu^{p}(T)=\inf \left\|\left(y_{n}\right)\right\|_{p} \cdot\left\|\left(x_{n}^{*}\right)\right\|_{p^{\prime}}^{w}
$$

where the infimum is taken over all representations of $T$ as above (see [10]). We will make use of the following theorem:

Theorem ([10, Theorem 1]). Let $p \in[1, \infty], T \in \mathcal{L}(X, Y)$ and suppose that either $X^{*}$ or $Y^{* * *}$ has the approximation property. If $T \in \mathcal{N}^{p}\left(X, Y^{* *}\right)$, then $T \in \mathcal{N}^{p}(X, Y)$. In other words, under these conditions, the p-nuclearity of $T^{*}$ implies that $T \in \mathcal{N}^{p}(X, Y)$.

Let $K$ be a bounded subset of $X$. We define the following bounded operators:

$$
\begin{aligned}
& u_{K}: \ell_{1}(K) \rightarrow X, \quad\left(\xi_{x}\right)_{x \in K} \mapsto \sum_{x \in K} \xi_{x} x, \\
& j_{K}: X^{*} \rightarrow \ell_{\infty}(K), \quad x^{*} \mapsto\left(\left\langle x, x^{*}\right\rangle\right)_{x \in K} .
\end{aligned}
$$

Notice that $u_{K}^{*}=j_{K}$. We write $u_{X}$ and $j_{X}$ instead of $u_{B_{X}}$ and $j_{B_{X}}$, respectively.

Proposition 3.5. Let $K$ be a bounded subset of $X$. The following statements are equivalent: 
(a) $K$ is relatively $p$-compact.

(b) $u_{K}$ is p-compact.

(c) $j_{K}$ is p-nuclear.

Proof. $(\mathrm{a}) \Leftrightarrow(\mathrm{b})$. This follows from the inclusions $K \subseteq u_{K}\left(B_{\ell_{1}(K)}\right) \subseteq$ $\overline{\mathrm{CO}}(K)$.

(b) $\Leftrightarrow(\mathrm{c})$. Let $u_{K}$ be $p$-compact. By Corollary $3.4, j_{K}$ is quasi $p$-nuclear, and since $\ell_{\infty}(K)$ is an injective space, $j_{K}$ is $p$-nuclear [6, Theorem 38]. For the converse, suppose $j_{K}$ is $p$-nuclear. According to [10, Theorem 1], the operator $u_{K}$ belongs to $\mathcal{N}^{p}\left(\ell_{1}(K), X\right)$ and, a fortiori, it is $p$-compact.

Corollary 3.6. Le $K$ be a subset of $X$. If $K$ is relatively p-compact in $X^{* *}$, then $K$ is p-compact in $X$. In particular, an operator $T \in \mathcal{L}(X, Y)$ is p-compact iff $T^{* *}$ is p-compact.

Proof. By Proposition 3.5, $J_{K}: x^{* * *} \in X^{* * *} \mapsto\left(\left\langle x, x^{* * *}\right\rangle\right)_{x \in K} \in \ell_{\infty}(K)$ is $p$-nuclear, hence so is $j_{K}=\left.J_{K}\right|_{X^{*}}: x^{*} \in X^{*} \mapsto\left(\left\langle x, x^{*}\right\rangle\right)_{x \in K} \in \ell_{\infty}(K)$. Again a call to Proposition 3.5 tells us that $K$ is $p$-compact in $X$.

REMARK 3.7. Let $A$ be a bounded subset of $X^{*}$. As in the proof of Proposition 3.5. $A$ is relatively $p$-compact iff the operator $\hat{\jmath}_{A}: x \in X \mapsto$ $\left(\left\langle x, x^{*}\right\rangle\right)_{x^{*} \in A} \in \ell_{\infty}(A)$ is $p$-nuclear.

In Corollary 3.4 it is shown that $\mathcal{K}_{p} \subseteq Q \mathcal{Q N}_{p}^{d}$. Now if $T \in \mathcal{L}(X, Y)$ is such that $T^{*} \in \mathcal{Q} \mathcal{N}_{p}\left(Y^{*}, X^{*}\right)$ then $T^{* *} \in \mathcal{K}_{p}\left(X^{* *}, Y^{* *}\right)$ (Corollary 3.4). From the above result, it follows that $T \in \mathcal{K}_{p}(X, Y)$. This leads to the following proposition which improves Proposition 5.3 in [13].

Proposition 3.8. $\mathcal{K}_{p}=2 \mathcal{N}_{p}^{d}$.

In a recent paper [14, Sinha and Karn have dealt with the Banach operator ideals $\mathcal{K}_{p}^{d}$ and $\mathcal{K}_{p}^{d d}$. The above results simplify the understanding of that paper, since $\mathcal{K}_{p}^{d}=\mathcal{Q \mathcal { N }}_{p}$ and $\mathcal{K}_{p}^{d d}=\mathcal{K}_{p}$.

Corollary 3.9. An operator $T \in \mathcal{L}(X, Y)$ is such that $T^{*} \in Q \mathcal{N}_{p}\left(Y^{*}, X^{*}\right)$ if and only if there exists $\left(y_{n}\right) \in \ell_{p}(Y)$ such that $\left\|T^{*} y^{*}\right\| \leq\left(\sum_{n}\left|\left\langle y_{n}, y^{*}\right\rangle\right|^{p}\right)^{1 / p}$ for all $y^{*} \in Y^{*}$.

As we have mentioned in the introduction, $p$-compact operators have been characterized as those operators whose adjoints factor through a subspace of $\ell_{p}$ [13, Theorem 3.1]. This factorization yields a complete norm defined on $\mathcal{K}_{p}(X, Y)$. Having in mind the preceding results, we have obtained the same factorization for the adjoints of $p$-compact operators in a much simpler way. In fact, Theorem 3.1 in [13] can be stated in the following manner:

Proposition 3.10. Let $X$ and $Y$ be Banach spaces and $p \in[1, \infty)$. The following statements are equivalent: 
(a) $T \in \mathcal{K}_{p}(X, Y)$.

(b) There exists a closed subspace $H$ of $\ell_{p}$ and operators $R \in Q \mathcal{N}_{p}\left(Y^{*}, H\right)$ and $S \in \mathcal{L}\left(H, X^{*}\right)$ such that $T^{*}=S \circ R$.

Proof. (a) $\Leftrightarrow(\mathrm{b})$. If $T \in \mathcal{K}_{p}(X, Y)$, there exists a sequence $\left(y_{n}\right) \in \ell_{p}(Y)$ such that $\left\|T^{*} y^{*}\right\| \leq\left(\sum_{n}\left|\left\langle y_{n}, y^{*}\right\rangle\right|^{p}\right)^{1 / p}$ for all $y^{*} \in Y^{*}$ (Proposition 3.1). Put

$$
H=\overline{\left\{\left(\left\langle y_{n}, y^{*}\right\rangle\right): y^{*} \in Y^{*}\right\}}
$$

and define the operators $R: y^{*} \in Y^{*} \mapsto\left(\left\langle y_{n}, y^{*}\right\rangle\right) \in H$ and $S:\left(\left\langle y_{n}, y^{*}\right\rangle\right) \in$ $H \mapsto T^{*} y^{*} \in Y^{*}$. It is easy to check that $H, R$ and $S$ satisfy the required conditions. The converse is trivial via Proposition 3.8 .

If $T \in \mathcal{K}_{p}(X, Y)$, we define

$$
k_{p}(T)=\inf \left\|\left(y_{n}\right)\right\|_{p}
$$

where the infimum is taken over all sequences $\left(y_{n}\right) \in \ell_{p}(Y)$ satisfying

$$
T\left(B_{X}\right) \subseteq\left\{\sum_{n} \alpha_{n} y_{n}:\left(\alpha_{n}\right) \in B_{\ell_{p^{\prime}}}\right\} .
$$

The inequality $k_{p}(T) \geq \nu_{p}^{Q}\left(T^{*}\right)$ (respectively, the equality $k_{p}\left(T^{*}\right)=\nu_{p}^{Q}(T)$ ) is a direct consequence of Proposition 3.1 (respectively, Proposition 3.2). Now, $\left[\mathcal{K}_{p}, k_{p}\right]$ becomes a Banach ideal and the proof is similar to that in [6, p. 31] showing that $\left[Q \mathcal{N}_{p}, \nu_{p}^{Q}\right]$ is a Banach ideal (both proofs can be connected via Proposition 3.1). According to [7, Theorem 6.1.8], the norm $k_{p}$ is equivalent to the norm $\kappa_{p}$ defined by Sinha and Karn in [13. Moreover, at the end of this section we prove that these norms coincide (Proposition 3.15).

Proposition 3.11. $\left[\mathcal{K}_{p}, k_{p}\right]$ is the surjective hull of $\left[\mathcal{N}^{p}, \nu^{p}\right]$ for all $p \in$ $[1, \infty)$

Proof. If $T \in \mathcal{L}(X, Y)$ and $T \circ u_{X}\left(B_{X}\right)\left[\ell_{1}\left(B_{X}\right) \stackrel{u_{X}}{\rightarrow} X \stackrel{T}{\rightarrow} Y\right]$ is relatively $p$-compact, then so is $T\left(B_{X}\right)$. In other words, $\mathcal{K}_{p}$ is surjective, and since $\mathcal{N}^{p} \subseteq \mathcal{K}_{p}$, we have $\left(\mathcal{N}^{p}\right)^{s} \subseteq \mathcal{K}_{p}$.

On the other hand, if $T \in \mathcal{K}_{p}(X, Y)$, then $T^{*} \in \mathcal{Q \mathcal { N }}_{p}\left(Y^{*}, X^{*}\right)$ (Corollary 3.4). Thus, $j_{X} \circ T^{*} \in Q \mathcal{N}_{p}\left(Y^{*}, \ell_{\infty}\left(B_{X}\right)\right)=\mathcal{N}_{p}\left(Y^{*}, \ell_{\infty}\left(B_{X}\right)\right)$, and since $j_{X} \circ T^{*}=\left(T \circ u_{X}\right)^{*}$ and $\ell_{\infty}\left(B_{X}\right)$ has the approximation property, it follows that $T \circ u_{X} \in \mathcal{N}^{p}\left(\ell_{1}\left(B_{X}\right), Y\right)$ ([10, Theorem 1]). So, we have obtained the equality $\left(\mathcal{N}^{p}\right)^{s}=\mathcal{K}_{p}$.

Now, a standard argument shows that

$$
\left(\mathcal{N}^{p}\left(\ell_{1}(I), Y\right), \nu^{p}\right)=\left(\mathcal{K}_{p}\left(\ell_{1}(I), Y\right), k_{p}\right) \quad \text { (isometrically) }
$$

for all nonempty sets $I$. In particular, this proves that $k_{p}=\left(\nu^{p}\right)^{s}$. 
Now, we can state our main result. We will need the following theorem:

Theorem ([11, Proposition 6.14]). Let $1 \leq p<\infty$ and let $X$ and $Y$ be Banach spaces. An operator $T: X \rightarrow Y$ is p-summing if and only if there exists a positive constant $C$ such that for every finite-dimensional subspace $E$ of $X$ and every finite-codimensional subspace $F$ of $Y$, the finite-dimensional operator

$$
q_{F} \circ T \circ i_{E}: E \rightarrow X \rightarrow Y \rightarrow Y / F
$$

satisfies $\pi_{p}\left(q_{F} \circ T \circ i_{E}\right) \leq C$. Furthermore, we have $\pi_{p}(T)=\inf C$, where the infimum is taken over all such pairs $E, F$.

Theorem 3.12. Let $T \in \mathcal{L}(X, Y)$ and $p \in[1, \infty)$. The following statements are equivalent:

(a) $T$ is p-summing.

(b) $T^{*}$ maps relatively compact subsets of $Y^{*}$ to relatively $p$-compact subsets of $X^{*}$.

Proof. (a) $\Rightarrow(\mathrm{b})$. Let $\left(y_{n}^{*}\right)$ be a null sequence in $Y^{*}$ and define $S: y \in$ $Y \mapsto\left(\left\langle y, y_{n}^{*}\right\rangle\right) \in c_{0}$. Obviously, $S$ is $\infty$-nuclear; therefore, $S \circ T$ is $p$-nuclear and

$$
\nu_{p}(S \circ T) \leq \nu_{\infty}(S) \pi_{p}(T) \leq \pi_{p}(T) \sup _{n}\left\|y_{n}^{*}\right\|
$$

[16, Theorem 9.13]. Then $(S \circ T)^{*}: e_{n} \in \ell_{1} \mapsto T^{*} y_{n}^{*} \in X^{*}$ belongs to $\mathcal{N}^{p}\left(\ell_{1}, X^{*}\right)$ and $\nu^{p}\left((S \circ T)^{*}\right) \leq \nu_{p}(S \circ T)$. As mentioned before, $\mathcal{K}_{p}\left(\ell_{1}, X^{*}\right)$ and $\mathcal{N}^{p}\left(\ell_{1}, X^{*}\right)$ are isometric, so

$$
k_{p}\left((S \circ T)^{*}\right) \leq \nu_{p}(S \circ T) \leq \pi_{p}(T) \sup _{n}\left\|y_{n}^{*}\right\| .
$$

This proves that the linear map

$$
U: c_{0}\left(Y^{*}\right) \rightarrow \mathcal{K}_{p}\left(\ell_{1}, X^{*}\right), \quad\left(y_{n}^{*}\right) \mapsto \sum_{n} e_{n}^{*} \otimes T^{*} y_{n}^{*},
$$

is well defined and $\|U\| \leq \pi_{p}(T)$ (this inequality will be used in the next proposition). Notice that, in particular, we have proved that the set $\left\{T^{*} y_{n}^{*}\right.$ : $n \in \mathbb{N}\}$ is relatively $p$-compact.

(b) $\Rightarrow($ a). To prove (a) we will use [11, Proposition 6.14]. Let $E$ be a finite-dimensional subspace of $X$ and $F$ a subspace of $Y$ whose codimension is finite. Given the sequence

$$
E \stackrel{i_{E}}{\rightarrow} X \stackrel{T}{\rightarrow} Y \stackrel{q_{F}}{\rightarrow} Y / F
$$

we obtain

$$
F^{\perp} \stackrel{q_{F}^{*}}{\rightarrow} Y^{*} \stackrel{T^{*}}{\rightarrow} X^{*} \stackrel{i_{E}^{*}}{\rightarrow} X^{*} / E^{\perp}
$$


For simplicity, we identify the operator $Q: e_{n} \in \ell_{1} \mapsto y_{n}^{*} \in Y^{*}$ with the sequence $\left(y_{n}^{*}\right)$. Now, consider the map

$$
\phi: \mathcal{K}\left(\ell_{1}, Y^{*}\right) \rightarrow \mathcal{K}_{p}\left(\ell_{1}, X^{*}\right), \quad\left(y_{n}^{*}\right) \mapsto\left(T^{*} y_{n}^{*}\right) .
$$

The map $\phi$ is linear and has closed graph, so it is continuous. Thus, there exists a positive constant $C$ such that $k_{p}\left(T^{*} y_{n}^{*}\right)<C$ for every relatively compact sequence $\left(y_{n}^{*}\right)$ in $B_{Y^{*}}$.

Choose $\left(y_{n}^{*}\right)$ dense in $B_{F^{\perp}}$. Since $k_{p}\left(T^{*} y_{n}^{*}\right)<C$, there exists a sequence $\left(x_{n}^{*}\right)$ in $\ell_{p}\left(X^{*}\right)$ such that $\left\|\left(x_{n}^{*}\right)\right\|_{p}<C$ and $\left\{T^{*} y_{n}^{*}\right\} \subset \overline{p \text {-co }\left(x_{n}^{*}\right)}$. By density, we also have $T^{*}\left(B_{F^{\perp}}\right) \subset \overline{p \text {-co }\left(x_{n}^{*}\right)}$. This yields $k_{p}\left(T^{*} \circ q_{F}^{*}\right) \leq\left\|\left(x_{n}^{*}\right)\right\|_{p}<C$ and therefore $k_{p}\left(i_{E}^{*} \circ T^{*} \circ q_{F}^{*}\right)<C$. Now, we can conclude that $k_{p}\left(i_{E}^{*} \circ T^{*}\right.$ $\left.\circ q_{F}^{*}\right)=\nu_{p}^{Q}\left(q_{F} \circ T \circ i_{E}\right)<C$ (see the comment after the definition of $k_{p}$ on page 297. Finally, recall that $\pi_{p} \leq \nu_{p}^{Q}$.

Proposition 3.13. Let $X, Y$ and $Z$ be Banach spaces and $p \geq 1$. If the operator $T: X \rightarrow Y$ is p-summing and $S: Z \rightarrow Y^{*}$ is compact, then $T^{*} \circ S$ is $p$-compact and $k_{p}\left(T^{*} \circ S\right) \leq \pi_{p}(T)\|S\|$.

Proof. Given $S \in \mathcal{K}\left(Z, Y^{*}\right)$ and $\varepsilon>0$ there exists a null sequence $\left(y_{n}^{*}\right)$ such that $S\left(B_{Z}\right) \subset \overline{\mathrm{co}}\left(y_{n}^{*}\right)$ and

$$
\sup _{n}\left\|y_{n}^{*}\right\|<\sup _{\|z\| \leq 1}\|S z\|+\varepsilon=\|S\|+\varepsilon .
$$

Now, we define the operator $A:\left(\alpha_{n}\right) \in \ell_{1} \mapsto \sum_{n} \alpha_{n} T^{*} y_{n}^{*} \in X^{*}$. In the above theorem we have proved that

$$
k_{p}(A) \leq \pi_{p}(T) \sup _{n}\left\|y_{n}^{*}\right\| .
$$

Thus, given $\delta>0$, there exists $\left(x_{n}^{*}\right)$ in $\ell_{p}\left(X^{*}\right)$ such that $\overline{\mathrm{co}}\left(T^{*} y_{n}^{*}\right) \subseteq$ $p$-co $\left(x_{n}^{*}\right)$ and $\left\|\left(x_{n}^{*}\right)\right\|_{p}<\pi_{p}(T)\left\|\left(y_{n}^{*}\right)\right\|_{\infty}+\delta$. Consequently, $T^{*}\left(S\left(B_{Z}\right)\right) \subseteq$ $\overline{\mathrm{co}}\left(T^{*} y_{n}^{*}\right) \subseteq p$-co $\left(x_{n}^{*}\right)$ and these inclusions yield

$$
k_{p}\left(T^{*} \circ S\right) \leq\left\|\left(x_{n}^{*}\right)\right\|_{p}<\pi_{p}(T)\left\|\left(y_{n}^{*}\right)\right\|_{\infty}+\delta .
$$

Letting $\delta \rightarrow 0$, we obtain $k_{p}\left(T^{*} \circ S\right) \leq \pi_{p}(T)\left\|\left(y_{n}^{*}\right)\right\|_{\infty}$. Finally, since $\left\|\left(y_{n}^{*}\right)\right\|_{\infty}$ $\leq\|S\|+\varepsilon$ we deduce

$$
k_{p}\left(T^{*} \circ S\right) \leq \pi_{p}(T)(\|S\|+\varepsilon) .
$$

The proof concludes by letting $\varepsilon \rightarrow 0$.

The dual version of the main theorem is also valid.

Theorem 3.14. Let $T \in \mathcal{L}(X, Y)$ and $p \in[1, \infty)$. The following statements are equivalent:

(a) $T^{*}$ is p-summing.

(b) $T$ maps relatively compact subsets of $X$ to relatively $p$-compact subsets of $Y$. 
Proof. (a) $\Rightarrow(\mathrm{b})$. This is an easy consequence of Theorem 3.12 and Corollary 3.6 .

(b) $\Rightarrow$ (a). By Proposition 3.5, we can consider the linear map

$$
V: c_{0}(X) \rightarrow \mathcal{N}_{p}\left(Y^{*}, \ell_{\infty}\right), \quad\left(x_{n}\right) \mapsto \sum_{n} T x_{n} \otimes e_{n}
$$

$\left(\left(e_{n}\right)\right.$ is the canonical basis of $\left.c_{0}\right)$. The operator $V$ is continuous because its graph is closed. Let $J$ be the restriction of $V^{*}$ to $\Pi_{p^{\prime}}\left(\ell_{\infty}, Y^{*}\right)$. A straightforward argument shows that $J: \Pi_{p^{\prime}}\left(\ell_{\infty}, Y^{*}\right) \rightarrow \ell_{1}\left(X^{*}\right)$ is the continuous linear map defined by $J(A)=\left(T^{*} \circ A\left(e_{n}\right)\right)$. As $\pi_{p^{\prime}}(A) \leq \nu_{p^{\prime}}(A)$ for all $A \in \mathcal{N}_{p^{\prime}}\left(\ell_{\infty}, Y^{*}\right)$, it follows that the map

$$
J_{0}: \mathcal{N}_{p^{\prime}}\left(\ell_{\infty}, Y^{*}\right) \rightarrow \ell_{1}\left(X^{*}\right), \quad A \mapsto\left(T^{*} \circ A\left(e_{n}\right)\right),
$$

is continuous. Now we consider $J_{0}^{*}: \ell_{\infty}\left(X^{* *}\right) \rightarrow \Pi_{p}\left(Y^{*}, \ell_{\infty}^{* *}\right)$ and $\phi=\left.J_{0}^{*}\right|_{c_{0}\left(X^{* *}\right)}$. If $\left(x_{n}^{* *}\right) \in c_{0}\left(X^{* *}\right), y^{*} \in Y^{*}$ and $\mu \in \ell_{\infty}^{*}$, then

$$
\begin{aligned}
\left\langle J_{0}^{*}\left(x_{n}^{* *}\right)\left(y^{*}\right), \mu\right\rangle & =J_{0}^{*}\left(x_{n}^{* *}\right)\left(\mu \otimes y^{*}\right)=\left\langle\left(x_{n}^{* *}\right), J_{0}\left(\mu \otimes y^{*}\right)\right\rangle \\
& =\left\langle\left(x_{n}^{* *}\right),\left(T^{*}\left[\mu \otimes y^{*}\left(e_{n}\right)\right]\right)\right\rangle=\sum_{n}\left\langle x_{n}^{* *}, T^{*}\left(\left\langle\mu, e_{n}\right\rangle y^{*}\right)\right\rangle \\
& =\sum_{n}\left\langle T^{* *} x_{n}^{* *}, y^{*}\right\rangle\left\langle\mu, e_{n}\right\rangle=\left\langle\sum_{n}\left\langle T^{* *} x_{n}^{* *}, y^{*}\right\rangle e_{n}, \mu\right\rangle .
\end{aligned}
$$

This proves that $\phi$ maps $c_{0}\left(X^{* *}\right)$ into $\Pi_{p}\left(Y^{*}, \ell_{\infty}\right)$ and $\phi\left(x_{n}^{* *}\right)=\sum_{n} T^{* *} x_{n}^{* *}$ $\otimes e_{n}$. Finally, we will show that $\phi\left(c_{0}\left(X^{* *}\right)\right) \subseteq \mathcal{N}_{p}\left(Y^{*}, \ell_{\infty}\right)$. First, for each $n \in \mathbb{N}$, we define

$$
\phi_{n}: \ell_{\infty}^{n}\left(X^{* *}\right) \rightarrow \Pi_{p}\left(Y^{*}, \ell_{\infty}^{n}\right), \quad\left(x_{k}^{* *}\right)_{k=1}^{n} \mapsto \sum_{k=1}^{n} T^{* *} x_{k}^{* *} \otimes e_{k} .
$$

By the ideal properties, we have $\left\|\phi_{n}\right\| \leq\|\phi\|$ for all $n \in \mathbb{N}$. In view of [16, Corollary 9.5], $\pi_{p}(u)=\nu_{p}(u)$ for all $u \in \mathcal{L}\left(Y^{*}, \ell_{\infty}^{n}\right)$. Thus, we can write (2) in the form

$$
\phi_{n}: \ell_{\infty}^{n}\left(X^{* *}\right) \rightarrow \mathcal{N}_{p}\left(Y^{*}, \ell_{\infty}^{n}\right), \quad\left(x_{k}^{* *}\right)_{k=1}^{n} \mapsto \sum_{k=1}^{n} T^{* *} x_{k}^{* *} \otimes e_{k} .
$$

Let us prove that $\left(\phi\left(x_{1}^{* *}, \ldots, x_{n}^{* *}, 0, \ldots\right)\right)_{n}$ is a Cauchy sequence in $\mathcal{N}_{p}\left(Y^{*}, \ell_{\infty}\right)$ for all $\left(x_{k}^{* *}\right) \in c_{0}\left(X^{* *}\right)$. According to (3) and the ideal properties of $\mathcal{N}_{p}$ we have

$$
\begin{aligned}
\nu_{p}\left(\phi \left(x_{1}^{* *}, \ldots,\right.\right. & \left.\left.x_{n}^{* *}, 0, \ldots\right)-\phi\left(x_{1}^{* *}, \ldots, x_{m}^{* *}, 0, \ldots\right)\right) \\
& =\nu_{p}\left(\phi\left(\ldots, 0, x_{m+1}^{* *}, \ldots, x_{n}^{* *}, 0, \ldots\right)\right) \leq\|\phi\| \cdot \sup _{m<k \leq n}\left\|x_{k}^{* *}\right\|
\end{aligned}
$$

for $n>m$. Thus, $\left(\phi\left(x_{1}^{* *}, \ldots, x_{n}^{* *}, 0, \ldots\right)\right)_{n}$ converges to an operator $S \in$ $\mathcal{N}_{p}\left(Y^{*}, \ell_{\infty}\right)$ and this operator is necessarily equal to $\phi\left(x_{n}^{* *}\right)=\sum_{n} T^{* *} x_{n}^{* *} \otimes e_{n}$. In particular, this implies that $T^{* *}$ maps relatively compact sets in $X^{* *}$ to 
relatively $p$-compact sets in $Y^{* *}$. Now, a call to Theorem 3.12 tells us that $T^{*}$ is $p$-summing.

We finish this section by showing that our definition of $k_{p}$ coincides with that in [13. An operator $T \in \mathcal{L}(X, Y)$ belongs to $\mathcal{K}_{p}(X, Y)$ if and only if there exists $\hat{y}=\left(y_{n}\right) \in \ell_{p}(Y)$ such that $T^{*}=S_{\hat{y}} \circ \phi_{\hat{y}}^{*}$, where $\phi_{\hat{y}}^{*}: y^{*} \in$ $Y^{*} \mapsto\left(\left\langle y_{n}, y^{*}\right\rangle\right) \in H:=\overline{\left\{\left(\left\langle y_{n}, y^{*}\right\rangle\right): y^{*} \in Y^{*}\right\}}$ and $S_{\hat{y}}:\left(\left\langle y_{n}, y^{*}\right\rangle\right) \in H \mapsto$ $T^{*} y^{*} \in X^{*}$ [13, Theorem 3.2]. Using this decomposition, we can endow $\mathcal{K}_{p}(X, Y)$ with the norm $\kappa_{p}$ defined by

$$
\kappa_{p}(T)=\inf \left\{\left\|S_{\hat{y}}\right\| \cdot\|\hat{y}\|_{p}: \hat{y}=\left(y_{n}\right) \in \ell_{p}(Y), T^{*}=S_{\hat{y}} \circ \phi_{\hat{y}}^{*}\right\} .
$$

Proposition 3.15. Let $X$ and $Y$ be Banach spaces and $p \geq 1$. Then $k_{p}(T)=\kappa_{p}(T)$ for all $T \in \mathcal{K}_{p}(X, Y)$.

Proof. Given $T \in \mathcal{K}_{p}(X, Y)$ and $\hat{y}=\left(y_{n}\right) \in \ell_{p}(Y)$, we know that $\left\|T^{*} y^{*}\right\| \leq\left\|\left(\left\langle y_{n}, y^{*}\right\rangle\right)\right\|_{p}$ for all $y^{*} \in Y^{*}$ if and only if $T\left(B_{X}\right) \subset p$-co $\left(y_{n}\right)$ (Proposition 3.1). Since $\left\|S_{\hat{y}}\left(\left\langle y_{n}, y^{*}\right\rangle\right)\right\|=\left\|T^{*} y^{*}\right\|$, it follows that $\left\|S_{\hat{y}}\right\| \leq 1$ and $\kappa_{p}(T) \leq k_{p}(T)$.

Now, given $0<\varepsilon<1$, consider $\hat{y}=\left(y_{n}\right) \in \ell_{p}(Y)$ such that

$$
\kappa_{p}(T)+\varepsilon>\left\|S_{\hat{y}}\right\| \cdot\|\hat{y}\|_{p} .
$$

Moreover, $\hat{y}$ can be chosen so that $\left\|S_{\hat{y}}\right\|>1-\varepsilon$. Otherwise, $\left\|T^{*} y^{*}\right\|=$ $\left\|S_{\hat{y}}\left(\left\langle y_{n}, y^{*}\right\rangle\right)\right\| \leq\left\|\left(\left\langle(1-\varepsilon) y_{n}, y^{*}\right\rangle\right)\right\|_{p}$ for all $y^{*} \in Y^{*}$ and this means that $T\left(B_{X}\right) \subset p-\operatorname{co}\left((1-\varepsilon) y_{n}\right)$ (Proposition 3.1). But then

$$
\begin{aligned}
\left\|S_{(1-\varepsilon) \hat{y}}\right\| & =\sup \left\{\left\|T^{*} y^{*}\right\|:\left\|\left(\left\langle(1-\varepsilon) y_{n}, y^{*}\right\rangle\right)\right\|_{p} \leq 1\right\} \\
& =\sup \left\{\left\|T^{*}\left(\frac{1}{1-\varepsilon} z^{*}\right)\right\|:\left\|\left(\left\langle(1-\varepsilon) y_{n}, \frac{1}{1-\varepsilon} z^{*}\right\rangle\right)\right\|_{p} \leq 1\right\} \\
& =\frac{\left\|S_{\hat{y}}\right\|}{1-\varepsilon},
\end{aligned}
$$

which implies $\kappa_{p}(T)+\varepsilon>\left\|S_{(1-\varepsilon) \hat{y}}\right\| \cdot\|(1-\varepsilon) \hat{y}\|_{p}$. By induction, we have $T\left(B_{X}\right) \subset p$-co $\left((1-\varepsilon)^{m} y_{n}\right)$ for all $m \in \mathbb{N}$, which is impossible if $T \neq 0$. So

$$
\kappa_{p}(T)+\varepsilon>(1-\varepsilon)\|\hat{y}\|_{p}>(1-\varepsilon) k_{p}(T),
$$

and since $\varepsilon$ can be chosen arbitrarily, $\kappa_{p}(T) \geq k_{p}(T)$.

4. The operator ideal $\mathcal{V}_{p}$. We will denote by $\mathcal{V}_{p}(X, Y)$ the vector space of all operators from $X$ into $Y$ that map relatively weakly $p$-compact subsets of $X$ to relatively $p$-compacts subsets of $Y$. In [13], the authors proved that $\Pi_{p}(X, Y) \subset \mathcal{V}_{p}(X, Y)$. First of all, we give sufficient conditions for which the converse inclusion holds for $p=1,2$. We will denote by $\ell_{p}^{u}(X)$ the subspace of $\ell_{p}^{w}(X)$ consisting of all unconditionally $p$-summable 
sequences in $X$, that is, those sequences $\left(x_{n}\right)$ satisfying

$$
\lim _{n \rightarrow \infty}\left(\sup _{\left\|x^{*}\right\| \leq 1} \sum_{m \geq n}\left|\left\langle x_{m}, x^{*}\right\rangle\right|^{p}\right)<\infty .
$$

Proposition 4.1. If $Y$ is an $\mathcal{L}_{1}$-space, then $\Pi_{1}(X, Y)=\mathcal{V}_{1}(X, Y)$ for every Banach space $X$.

Proof. If $\left(x_{n}\right) \in \ell_{1}^{u}(X)$ and $T \in \mathcal{V}_{1}(X, Y)$, then the set

$$
\left\{\sum_{n} \alpha_{n} T\left(x_{n}\right):\left(\alpha_{n}\right) \in B_{c_{0}}\right\}
$$

is relatively 1-compact in $Y$. So, the operator $A: e_{n} \in c_{0} \mapsto T\left(x_{n}\right) \in Y$ is 1-compact. By Corollary 3.4, its adjoint $A^{*}: Y^{*} \rightarrow \ell_{1}$ is quasi 1-nuclear, and therefore it is 1 -summing. As $Y^{*}$ is an $\mathcal{L}_{\infty}$-space, $A^{*}$ is integral. Actually, $A^{*}$ is nuclear because $\ell_{1}$ is a dual space and has the Radon-Nikodym property. According to [3, Theorem VIII.7], $A$ is nuclear. This yields $\sum_{n}\left\|T\left(x_{n}\right)\right\|$ $<\infty$.

Proposition 4.2. If $Y$ is a Banach space isomorphic to a Hilbert space, then $\Pi_{2}(X, Y)=\mathcal{V}_{2}(X, Y)$ for every Banach space $X$.

Proof. Let $T \in \mathcal{V}_{2}(X, Y)$ and $\left(x_{n}\right) \in \ell_{2}^{w}(X)$. By hypothesis, the operator $S: \ell_{2} \rightarrow Y$ defined by $S\left(e_{n}\right)=T\left(x_{n}\right)$ is 2-compact, and therefore its adjoint $S^{*}: y^{*} \in Y^{*} \mapsto\left(\left\langle T\left(x_{n}\right), y^{*}\right\rangle\right) \in \ell_{2}$ is quasi 2-nuclear (Corollary 3.4). According to [2, Theorem 4.19], $S^{*}$ has a 2-summing adjoint because $Y^{*}$ is isomorphic to a Hilbert space. In particular, $S$ is 2-summing and this implies that $\sum_{n}\left\|T\left(x_{n}\right)\right\|^{2}<\infty$. So, we have proved that $T$ is 2 -summing.

However, in general, $\Pi_{p}(X, Y)$ is strictly contained in $\nu_{p}(X, Y)$ for all $p \in[1, \infty)$. The following relationships are obvious for all $p \geq 1$ :

$$
\Pi_{p}\left(\ell_{p^{\prime}}, X\right) \subset \Phi_{p}\left(\ell_{p}(X)\right) \subset \mathcal{K}_{p}\left(\ell_{p^{\prime}}, X\right)=\mathcal{V}_{p}\left(\ell_{p^{\prime}}, X\right) .
$$

If $p>1$, the first inclusion is strict whenever $X$ is not a subspace of a quotient of an $L_{p}$-space [15, Theorem 3.1]. So, only the case $p=1$ needs to be studied.

Let $1 \leq p<2$. Let $\mathcal{C}_{p}$ be the ideal of all operators mapping weakly $p$-summable sequences to unconditionally $p$-summable sequences. First of all, we will prove that $\Pi_{p}^{d} \circ \mathcal{C}_{p} \subset \mathcal{V}_{p}$ for every $p \geq 1$. So, let $T=T_{2} \circ T_{1}$, where $T_{1}$ belongs to $\mathcal{C}_{p}(X, Y)$ and $T_{2} \in \Pi_{p}^{d}(Y, Z)$. If $\left(x_{n}\right)$ is a weakly $p$ summable sequence in $X$ and $A=\left\{\sum_{n} \alpha_{n} x_{n}:\left(\alpha_{n}\right) \in B_{\ell_{p^{\prime}}}\right\}$, notice that $T_{1}(A)$ is relatively compact in $Y$. Then $T_{2}\left(T_{1}(A)\right)$ is relatively $p$-compact (Theorem 3.12).

Now we are going to show that the inclusion $\Pi_{p} \subset V_{p}$ is, in general, strict for every $1 \leq p<2$. Denote by $I_{2,0}$ the identity map from $\ell_{2}$ into $c_{0}$. According to [1, Lemma 6] the identity map from $\ell_{2}$ onto $\ell_{2}$ belongs to $\mathcal{C}_{p}$ for 
every $p<2$. On the other hand, $\left(I_{2,0}\right)^{*}$ is $p$-summing, so $I_{2,0} \in \Pi_{p}^{d} \circ \mathrm{C}_{p} \subset \mathcal{V}_{p}$ for all $p<2$. Nevertheless, $I_{2,0}$ is not $p$-summing.

Finally, we have obtained the following result about the biadjoint of an operator $T \in \mathcal{V}_{2}$. Here, $\mathcal{J}_{p}$ denotes the Banach ideal of $p$-integral operators.

Proposition 4.3. Let $X$ be a Banach space such that $I_{X^{* *}} \in \mathcal{C}_{2}$. If $T \in \mathcal{V}_{2}(X, Y)$, then $T^{* *} \in \mathcal{V}_{2}\left(X^{* *}, Y^{* *}\right)$.

Proof. Given $T \in \mathcal{V}_{2}(X, Y)$, consider the linear map

$$
U:\left(x_{n}\right) \in \ell_{2}^{u}(X) \mapsto \sum_{n} T x_{n} \otimes e_{n} \in \mathcal{Q N}_{2}\left(Y^{*}, \ell_{2}\right) .
$$

It is easy to prove that $U$ has closed graph, and therefore it is continuous. Its adjoint maps $\mathcal{J}_{2}\left(\ell_{2}, Y^{* * *}\right)$ into $\mathcal{J}_{1}\left(\ell_{2}, X^{*}\right)$. Put $V=\left.U^{*}\right|_{\mathcal{N}_{2}\left(\ell_{2}, Y^{*}\right)}$. Since $\mathcal{N}_{1}\left(\ell_{2}, X^{*}\right)$ is isometric to a subspace of $\mathcal{J}_{1}\left(\ell_{2}, X^{*}\right)$ it follows easily that $V$ maps $\mathcal{N}_{2}\left(\ell_{2}, Y^{*}\right)$ into $\mathcal{N}_{1}\left(\ell_{2}, X^{*}\right)$. We also denote by $V$ the operator

$$
\sum_{n} e_{n}^{*} \otimes y_{n}^{*} \in \mathcal{N}_{2}\left(\ell_{2}, Y^{*}\right) \mapsto \sum_{n} e_{n}^{*} \otimes T^{*} y_{n}^{*} \in \mathcal{N}_{1}\left(\ell_{2}, X^{*}\right) .
$$

Taking adjoints again we obtain the operator

$$
\left(x_{n}^{* *}\right) \in \mathcal{L}\left(X^{*}, \ell_{2}\right) \stackrel{V^{*}}{\mapsto} \sum_{n} T^{* *} x_{n}^{* *} \otimes e_{n} \in \Pi_{2}\left(Y^{*}, \ell_{2}\right) .
$$

As every 2-summing operator is 2-integral and the 2-summing norm coincides with the 2-integral norm, (5) can be written in the form

$$
\left(x_{n}^{* *}\right) \in \mathcal{L}\left(X^{*}, \ell_{2}\right) \stackrel{V^{*}}{\mapsto} \sum_{n} T^{* *} x_{n}^{* *} \otimes e_{n} \in \mathcal{J}_{2}\left(Y^{*}, \ell_{2}\right) .
$$

Now, as in the proof of $(\mathrm{b}) \Rightarrow(\mathrm{a})$ in Theoren 3.14 , we can prove that $V^{*}$ maps $\ell_{2}^{u}\left(X^{* *}\right)$ into $\mathcal{N}_{2}\left(Y^{*}, \ell_{2}\right)$. This shows that the operator $A: y^{*} \in$ $Y^{*} \mapsto\left(\left\langle T^{* *} x_{n}^{* *}, y^{*}\right\rangle\right) \in \ell_{2}$ is 2-nuclear whenever $\left(x_{n}^{* *}\right)$ is unconditionally 2-summable in $X^{* *}$, and therefore its adjoint $A^{*}: e_{n} \in \ell_{2} \mapsto T^{* *} x_{n}^{* *} \in Y^{* *}$ belongs to $\mathcal{N}^{2}$. So, $A^{*}$ is 2 -compact and this concludes the proof.

Acknowledgments. This research was supported by MTM2009-14483C02-01 project (Spain).

\section{References}

[1] J. M. Castillo, On Banach spaces $X$ such that $\mathcal{L}\left(L_{p}, X\right)=\mathcal{K}\left(L_{p}, X\right)$, Extracta Math. 10 (1995), 27-36.

[2] J. Diestel, H. Jarchow and A. Tonge, Absolutely Summing Operators, Cambridge Stud. Adv. Math. 43, Cambridge Univ. Press, Cambridge, 1995.

[3] J. Diestel and J. J. Uhl, Vector Measures, Math. Surveys Monogr. 15, Amer. Math. Soc., Providence, RI, 1977. 
[4] A. Grothendieck, Produits tensoriels topologiques et espaces nucléaires, Mem. Amer. Math. Soc. 16 (1955).

[5] H. Jarchow, Locally Convex Spaces, Teubner, Stuttgart, 1981.

[6] A. Persson und A. Pietsch, p-nukleare und p-integrale Abbildungen in Banachräumen, Studia Math. 33 (1969), 19-62.

[7] A. Pietsch, Operator Ideals, North-Holland, 1978.

[8] O. I. Reinov, Approximation properties of order $p$ and the existence of non-p-nuclear operators with p-nuclear second adjoints, Math. Nachr. 109 (1982), 125-134.

[9] - Disappearance of tensor elements in the scale of $p$-nuclear operators, in: Operator Theory and Function Theory, no. 1, Leningrad. Univ., Leningrad, 1983, 145-165.

[10] - On linear operators with p-nuclear adjoint, Vestnik St. Petersburg Univ. Math. 33 (2000), no. 4, 19-21.

[11] R. Ryan, Introduction to Tensor Products of Banach Spaces, Springer, London, 2002.

[12] P. D. Saphar, Hypothèse d'approximation à l'ordre $p$ dans les espaces de Banach et approximation d'applications p absolument sommantes, Israel J. Math. 13 (1972), 379-399.

[13] D. P. Sinha and A. K. Karn, Compact operators whose adjoints factor through subspaces of $\ell_{p}$, Studia Math. 150 (2002), 17-33.

[14] —, - Compact operators which factor through subspaces of $\ell_{p}$, Math. Nachr. 3 (2008), 412-423.

[15] Y. Takahashi and Y. Okazaki, Characterization of subspaces, quotients and subspaces of quotients of $L_{p}$, Hokkaido Math. J. 15 (1986), 233-241.

[16] N. Tomczak-Jaegermann, Banach-Mazur Distances and Finite-Dimensional Operator Ideals, Longman Sci. Tech., London, 1989.

J. M. Delgado, C. Piñeiro, E. Serrano

Departamento de Matemáticas

Campus Universitario del Carmen

Universidad de Huelva

Avda. de las Fuerzas Armadas s/n

21071 Huelva, Spain

E-mail: jmdelga@uhu.es

candido@uhu.es

eserrano@uhu.es 